\title{
Actual Optical and Thermal Performance of Photovoltaic Modules
}

\author{
Hamdy K. Elminir, V. Benda, I. Kudláček
}

Field testing is costly, time-consuming and depends heavily on prevailing weather conditions. Adequate security and weather protection must also be provided at the test site. Delays can be caused due to bad weather and system failures. To overcome these problems, a photovoltaic array simulation may be used. In any simulation scheme involving photovoltaic systems, one important choice is the selection of a mathematical model.

In the literature several approaches to the problem have been made. Most procedures designed for this purpose are based on analytical descriptions of the physical mechanisms inside the solar cell that can be represented by a circuit diagram with discrete components, like a two-exponential model. Such simulators have some merits. However, their limited flexibility in readily simulating the influence of solar radiation, temperature and various array parameters is a serious drawback that has been noted. To get more accurate results in predicting the actual performance of photovoltaic modules, the parameters influencing incoming (optical parameters) and outgoing power flow (electrical and thermal parameters) were investigated by simulation and by some verifying experiments, to get a closer insight into the response behavior of this element, and to estimate the overall performance as well as optimization of the parameters.

Keywords: solar radiation, reflection losses, radiation shape factor, radiative surface area, temperature distribution, emissivity, isotropic model, Hay and Klucher's anisotropic models.

\section{Introduction}

Design and performance evaluation of photovoltaic systems usually involve an estimation of irradiation incident on the photovoltaic module plane. For design purposes only gloal horizontal irradiation, published by meteorological institutes, is usually available. Using irradiation models, we can calculate the gain in irradiation on a tilted plane with respect to horizontal irradiation. Concerning a part of this task, most textbooks [4] recommend that the diffuse component be treated as if it were isotropically emanating from the sky vault. However, theoretical as well as experimental results have shown that this simplifying assumption is generally far from reality. Dave, J., 1977 examined the validity of this isotropic distribution approximation for a sun-facing flat surface located at the bottom of plane parallel models of non-absorbing homogeneous atmospheres. Even for such idealized and somewhat unrealistic models, he showed that the use of an isotropic distribution approximation results in systematic underestimation of the diffuse energy contribution to the sun-facing surfaces. That study demonstrated the need to test this approximation for more realistic, atmospheric conditions. Thus it appears that sky radiance should be treated as anisotropic, particularly because of the strong forward scattering effect of aerosols [2, 9, 14, 16].

In the first part of this study, some results concerning the accuracy of models to estimate irradiance on inclined planes are tested. The models chosen for discussion here are for arbitrary sky conditions and are supposed to be applicable anywhere in the world. These methods begin with measured hourly global and diffuse radiation received on a horizontal surface. These quantities are then transposed onto an inclined plane by a mathematical procedure. The accuracies of the models are then compared on the basis of statistical error tests, and the most accurate model is recommended. The analyses are also extended to include the optical processes occurring inside the encapsulation solar module.

Solar concentrators are frequently used to increase the specific power and decrease the space of radiation degrada- tion of solar cell parameters. On the other hand, at high light concentration coefficients, a large amount of thermal energy evolves in solar cells. If cooling is not provided, the cell working temperature increases. This affects the carrier concentration and light absorption process, causing a reduction in open circuit voltage, a slight increase in short circuit current, a reduction of the fill factor, and degradation of the cell power output. The degradation rate of cell output is approximately double for each $10^{\circ} \mathrm{C}$ increase in temperature $[7,22]$. Therefore, the modules and solar array must take full advantage of radiative, conductive and convective cooling and absorb the minimum of unused radiation. Hence, there is a need for an exact technique to calculate accurately and efficiently the temperature distribution of photovoltaic solar modules, from which we can adjust safe and proper operation at maximum ratings. The computer code was written in Fortran, a programming language quite flexible and suitable for organizing the screen with multiple windows and for drawing curves and histograms. The program structure consists of a main executable file, which has several external units to perform the different calculations; program maintenance is therefore quite easy, as is the implementation of new models or extension to a new configuration. The derived equations are solved using an explicit technique and iteration processes [18, 20]. The models and simulation programs developed here allow us to predict the thermal performance, and they are promising tools for evaluating new photovoltaic power plants with the aim of increasing efficiency. Three main criteria are considered in evaluating the models. The first is versatility: What range of photovoltaic modules and systems can the model handle? Accuracy is the second issue: What is the physical basis and how closely can the model replicate manufacturers' data? The final issue is computational speed. This criterion is of secondary importance for this work.

\section{Apparatus and measurements}

Total radiation was monitored with a high precision pyranometer, which is sensitive in the wavelength range from 300 to $3000 \mathrm{~nm}$. Sky diffuse radiation was measured by 
a pyranometer equipped with a special shading device to exclude direct radiation from the sun. All data sets were subjected to various quality control tests. Three types of data checks were performed to identify missing data, data that clearly violates physical limits, and extreme data. Hours when the data was known to be "bad" or "missing" were omitted. Second, any hour with an observation that violated a physical limit or conservation principle was eliminated from the data set, including: reported hours with a diffuse fraction greater than 1, or beam radiation exceeding the extraterrestrial beam radiation. To eliminate the uncertainty associated with radiation measurements at large incidence angles, hours with a zenith angle larger than $80^{\circ}$ were eliminated. The final data set was constructed from the measured data that passed all of the quality control checks.

\section{Optical performance of photovoltaic modules}

In order to make a precise representation of the actual optical conditions in the module, a model for an encapsulating cell was developed which determines the insolation reaching the cell from sun and sky irradiance. This was done by modeling the optical processes occurring outside and inside the encapsulation. Most collecting devices associated with solar energy systems are tilted at some angle with respect to the horizontal. Due to the lack of measured tilted surface solar radiation data, models are employed to estimate the radiation incident on a collector surface from measured horizontal radiation. In the present work, methods for calculating radiation on a tilted surface from horizontal data are evaluated.

\subsection{Evaluation of irradiation on a tilted south-oriented surface}

Consider a plane inclined at an angle $\beta$ from the horizontal position. For the time being, we assume that the surface is inclined in such a way that it faces the sun; that is, direct radiation is not striking the back of this plane. Total radiation arriving on an inclined surface is composed of three components: beam radiation, sky diffuse radiation and radiation reflected from the ground. The models discussed here all share the same formulations for the beam and ground reflected components. Hourly irradiations with a solar elevation angle $\gamma>8^{\circ}$ are tested only in order to avoid errors resulting from dividing small numbers. It is useful to write down the hourly formulation of these components.

\subsubsection{Beam radiation incident on an inclined surface}

For a surface oriented in any direction with respect to the meridian, the trigonometric relation for the incidence angle, $\theta_{\mathrm{I}}$ can be written in the following form:

$$
\begin{aligned}
\cos \theta_{\mathrm{I}}= & (\sin \phi \cos \beta-\cos \phi \sin \beta \cos \gamma) \sin \delta+ \\
& +(\cos \phi \cos \beta+\sin \phi \sin \beta \cos \gamma) \cos \delta \cos \omega+ \\
& +\cos \delta \sin \beta \sin \gamma \sin \omega
\end{aligned}
$$

where the meanings of the different symbols are as given in the nomenclature list. There are several commonly occurring cases for which Equation 1 is simplified. For a fixed surface sloped toward the south or north, that is, with a surface azimuth angle $\gamma$ of $0^{\circ}$ or $180^{\circ}$ (a very common situation for fixed flat plate collectors), the last term drops out.

$$
\begin{aligned}
\cos \theta_{\mathrm{I}}= & (\sin \phi \cos \beta-\cos \phi \sin \beta) \sin \delta+ \\
& +(\cos \phi \cos \beta+\sin \phi \sin \beta) \cos \delta \cos \omega= \\
& =\sin \delta \sin (\phi-\beta)+\cos \delta \cos \omega \cos (\phi-\beta) .
\end{aligned}
$$

For horizontal surfaces, the angle of incidence, $\theta_{\mathrm{I}}$ is the zenith angle of the sun, $\theta_{\mathrm{z}}$. Its value must be between $0^{\circ}$ and $90^{\circ}$ when the sun is above the horizon. For this situation $\beta=0$, and Equation 1 becomes:

$$
\cos \theta_{Z}=\cos \phi \cos \delta \cos \omega+\sin \phi \sin \delta .
$$

The geometric factor $R_{\mathrm{b}}$, the ratio of beam radiation on the tilted surface, $I_{\mathrm{b}, \mathrm{T}}$ to that on a horizontal surface at any time, can be calculated exactly by appropriate use of Equation 1 .

The ratio $I_{\mathrm{b}, \mathrm{T}} / I_{\mathrm{b}, \mathrm{H}}$ is given by:

$$
R_{\mathrm{b}}=\frac{\cos \theta_{\mathrm{I}}}{\cos \theta_{\mathrm{Z}}}=\frac{\sin \delta \sin (\phi-\beta)+\cos \delta \cos \omega \cos (\phi-\beta)}{\cos \phi \cos \delta \cos \omega+\sin \phi \sin \delta} .
$$

The hourly beam radiation received on an inclined surface can be expressed as:

$$
I_{\mathrm{b}, \mathrm{T}}=I_{\mathrm{b}, \mathrm{H}} \cdot R_{\mathrm{b}}=\left(G_{\mathrm{H}}-I_{\mathrm{d}, \mathrm{H}}\right) \cdot R_{\mathrm{b}} .
$$

It should be noted that at the grazing angles (just at sunrise or at sunset) $R_{\mathrm{b}}$ can change rapidly and may approach infinity or zero because both the numerator and the denominator are small numbers. This depends on slope, latitude and date.

\subsubsection{Sky diffuse radiation incident on an inclined surface}

Diffuse irradiance is difficult to determine accurately with the simple parameterization methods that were used to calculate direct normal irradiance in the previous section, since its spatial distribution is generally unknown and time dependent. Three diffuse subcomponents are used to approximate the anisotropic behavior of diffuse radiation. The first is an isotropic part received uniformly from the entire sky dome. The second is circumsolar diffuse resulting from forward scattering of solar radiation and concentrated in the part of the sky around the sun. The third, referred to as horizon brightening, is concentrated near the horizon, and is most pronounced in clear skies. Several models have been proposed to estimate the diffuse radiation on a tilted surface (not all of which account for these three diffuse subcomponents).

The Isotropic model [12] is the simplest of the tilted surface models. In this model the intensity of sky diffuse radiation is assumed uniform over the sky dome, i.e., it is independent of the azimuth and zenith angles. It approximates the completely overcast sky condition. The formula for the hourly sky diffuse radiation incident on an inclined plane is given by the product of the hourly diffuse radiation incident on a horizontal surface and the configuration factor from the surface to the sky, $(1+\cos \beta) / 2$.

$$
I_{\mathrm{d}, \mathrm{T}}=I_{\mathrm{d}, \mathrm{H}} \frac{1+\cos \beta}{2} \text {. }
$$

Under completely cloudy skies, the isotropic model becomes a good approximation. As skies become clearer, the validity of the isotropic sky model deteriorates due to the presence of circumsolar and horizon brightening anisotropic effects.

Klucher, T., 1979 model: This model is based on a study of clear sky conditions by Temps, R. and Coulson, K., 1977. Their model was modified by Klucher, who incorporated 
conditions of cloudy skies. It takes into account the increase in the diffuse radiation in the vicinity of the sun (circumsolar radiation) and that near the horizon (horizon brightening). Klucher's formulation of the hourly sky diffuse radiation incident on an inclined surface is:

$I_{\mathrm{d}, \mathrm{T}}=I_{\mathrm{d}, \mathrm{H}}\left[\left(\frac{1+\cos \beta}{2}\right)\left(1+F_{1} \sin ^{3}\left(\frac{\beta}{2}\right)\right)\left(1+F_{1} \cos ^{2} \theta_{\mathrm{Z}} \sin ^{3} \theta_{\mathrm{Z}}\right)\right] .(7$ )

Where $F_{1}$ is the modulating function given by:

$$
F_{1}=1-\left(\frac{I_{\mathrm{d}, \mathrm{H}}}{G_{\mathrm{H}}}\right)^{2} \text {. }
$$

When the skies are completely overcast, $F_{1}=0$, Klucher's model reverts to the isotropic model.

Hay, J. and Davies, J., 1980 developed a model to predict tilted surface diffuse radiation, which accounts for both circumsolar and isotropic diffuse radiation. Realizing that the anisotropic behavior of circumsolar diffuse radiation becomes more pronounced under clear sky conditions, Hay, J. and Davies, J., 1980 defined an "anisotropic index, $A_{\mathrm{I}}$ " to weight the circumsolar, $I_{\mathrm{T} \text {, cir }}$ and isotropic, $I_{\mathrm{T}}$ iso radiation components. The anisotropy index defines a portion of the diffuse radiation to be treated as circumsolar with the remaining portion to be considered isotropic. The circumsolar diffuse radiation is projected onto the tilted surface in the same fashion as beam radiation.

$$
\begin{aligned}
& A_{\mathrm{I}}=I_{\mathrm{b}, \mathrm{H}} / I_{\mathrm{O}} \\
& I_{\mathrm{T}, \text { cir }}=I_{\mathrm{d}, \mathrm{H}} \cdot A_{\mathrm{I}} \cdot R_{\mathrm{b}} .
\end{aligned}
$$

The remaining diffuse radiation is treated as isotropic diffuse.

$$
I_{\mathrm{T}, \text { iso }}=I_{\mathrm{d}, \mathrm{H}}\left(1-A_{\mathrm{I}}\right) \frac{1+\cos \beta}{2} .
$$

The total diffuse radiation on a tilted surface is the sum of $(10)$ and (11).

$$
I_{\mathrm{d}, \mathrm{T}}=I_{\mathrm{d}, \mathrm{H}}\left[\left(1-A_{\mathrm{I}}\right)\left(\frac{1+\cos \beta}{2}\right)+A_{\mathrm{I}} \cdot R_{\mathrm{b}}\right] .
$$

Under clear skies, the anisotropy index will be high and the circumsolar diffuse is weighted more heavily than the isotropic diffuse. Under cloudy skies, the anisotropy index goes to zero and all diffuse is treated as isotropic. The Hay model does not account for horizon brightening radiation incident on south facing surfaces.

\subsubsection{Ground reflected radiation incident on an inclined surface}

In order to correctly evaluate the diffuse radiation incident on an inclined surface of any orientation and of any tilt angle, we need to known separately the sky diffuse radiation as well as the ground reflected radiation. In this section we are going to focus on ground reflected radiation, which is also significant and which can sometimes reach values of the order of $100 \mathrm{~W} / \mathrm{m}^{2}$ for a vertical plane. It has already been previously found, but based on restricted data, that using an albedo value measured on site and considered as constant leads to satisfactory results, Ineichen et al., 1987.

$$
I_{\mathrm{g}, \mathrm{H}}=\rho(\text { site }) \cdot G_{\mathrm{H}} \text {. }
$$

A common method for calculating the ground reflected radiation incident on a tilted surface is to assume that the foreground in the collector field of view is a diffuse reflector and that the horizon is unobstructed. Other authors have proposed anisotropic ground reflectance models [6, 8], but lack of experimental data has hampered their validation. Therefore, the ground reflected radiation is assumed to be diffuse. Under the isotropic condition, the ground reflected radiation incident on the inclined surface is given by the above quantity multiplied by the configuration factor from the ground to the inclined surface. Thus:

$$
I_{\mathrm{g}, \mathrm{T}}=\frac{1}{2} G_{\mathrm{H}} \rho(1-\cos \beta) .
$$

\subsubsection{Model evaluation}

Three irradiance models are considered in this work: the isotropic model, Hay's and Klucher's anisotropic models. For each model, measured diffuse and horizontal global values were used to calculate the irradiance on surfaces tilted at $30^{\circ}$, $60^{\circ}$ and $90^{\circ}$ above the horizon. The results were compared with the irradiances monitored and presented in terms of usual statistics: mean bias error (MBE) and root mean square error (RMSE). The results for south facing surfaces are given in Table 1 and Table 2. It is noted that the RMSEs for all three models increase as the slope of the collector increases, but remain in a domain of errors for which these relations can

Table 1: Root mean square errors [\%] for global radiation received on inclined surfaces

\begin{tabular}{|l|c|c|c|c|c|c|c|c|c|}
\hline \multirow{2}{*}{ Month } & \multicolumn{3}{c|}{ Isotropic Model RMSE } & \multicolumn{3}{c|}{ Hay's Model RMSE } & \multicolumn{3}{c|}{ Klucher's Model RMSE } \\
\cline { 2 - 12 } & $30^{\circ}$ & $60^{\circ}$ & $90^{\circ}$ & $30^{\circ}$ & $60^{\circ}$ & $90^{\circ}$ & $30^{\circ}$ & $60^{\circ}$ & $90^{\circ}$ \\
\hline January & 18.0 & 22.0 & 22 & 8.0 & 11.0 & 10.0 & 8.0 & 11.0 & 13 \\
\hline February & 16.0 & 21.0 & 22 & 7.5 & 11.0 & 12.0 & 7.0 & 11.0 & 13 \\
\hline March & 9.0 & 12.0 & 12 & 4.0 & 6.0 & 8.5 & 3.0 & 5.0 & 8 \\
\hline April & 6.0 & 14.0 & 8 & 4.0 & 12.0 & 8.0 & 3.0 & 12.0 & 8 \\
\hline May & 3.5 & 5.5 & 12 & 3.0 & 5.5 & 12.0 & 3.0 & 5.0 & 8 \\
\hline June & 4.0 & 9.0 & 25 & 3.0 & 9.0 & 21.0 & 4.5 & 8.0 & 24 \\
\hline July & 3.5 & 6.0 & 19 & 2.0 & 5.0 & 13.0 & 4.0 & 6.5 & 18 \\
\hline August & 3.0 & 7.0 & 12 & 2.0 & 5.0 & 10.0 & 3.0 & 5.0 & 12 \\
\hline September & 11.0 & 15.0 & 17 & 6.5 & 9.0 & 12.0 & 4.5 & 7.0 & 10 \\
\hline October & 13.0 & 16.0 & 17 & 6.5 & 7.0 & 9.0 & 5.0 & 6.5 & 9 \\
\hline November & 24.0 & 31.0 & 36 & 13.0 & 8.0 & 20.0 & 10.0 & 14.0 & 19 \\
\hline December & 18.0 & 23.0 & 26 & 9.0 & 13.0 & 14.0 & 9.0 & 11.0 & 13 \\
\hline
\end{tabular}


Table 2: Mean bias errors [\%] for global radiation received on inclined surfaces

\begin{tabular}{|l|c|c|c|c|c|c|c|c|c|}
\hline \multirow{2}{*}{ Month } & \multicolumn{3}{c|}{ Isotropic Model MBE } & \multicolumn{3}{c|}{ Hay's Model MBE } & \multicolumn{3}{c|}{ Klucher's Model MBE } \\
\cline { 2 - 11 } & $30^{\circ}$ & $60^{\circ}$ & $90^{\circ}$ & $30^{\circ}$ & $60^{\circ}$ & $90^{\circ}$ & $30^{\circ}$ & $60^{\circ}$ & $90^{\circ}$ \\
\hline January & -8.3 & -10.3 & -9.2 & -2.2 & -1.6 & -1.2 & -3.3 & -3.9 & -4.2 \\
\hline February & -8.2 & -11.0 & -11.7 & -2.8 & -3.3 & -3.3 & -2.8 & -4.7 & -5.5 \\
\hline March & -5.5 & -6.1 & -4.4 & -1.7 & -0.6 & 1.0 & -0.2 & -0.1 & 1.7 \\
\hline April & -3.3 & -5.3 & -1.8 & -1.1 & -2.5 & -0.6 & -0.2 & -0.2 & 4.5 \\
\hline May & -1.7 & -2.2 & -2.4 & -1.2 & -2.2 & -5.5 & 1.6 & 2.2 & 4.8 \\
\hline June & -0.6 & -1.1 & 1.2 & -0.5 & -1.9 & -4.4 & 2.2 & 3.0 & 10.0 \\
\hline July & -0.8 & -0.6 & 1.1 & -0.4 & -1.1 & -3.9 & 2.2 & 4.7 & 8.9 \\
\hline August & -2.5 & -2.8 & -2.2 & -1.1 & -0.8 & -2.2 & 1.9 & 2.2 & 5.5 \\
\hline September & -4.4 & -4.4 & -3.6 & -2.2 & -1.1 & -0.6 & 0.1 & 1.1 & 3.2 \\
\hline October & -5.0 & -8.9 & -9.4 & -3.3 & -2.8 & -2.8 & -2.2 & -2.8 & 1.9 \\
\hline November & -8.3 & -9.4 & -10.0 & -4.4 & -2.6 & -2.2 & -3.3 & -2.9 & 0.8 \\
\hline December & -6.7 & -7.8 & -7.2 & -1.1 & -0.2 & 0.0 & -2.1 & -0.3 & -0.5 \\
\hline
\end{tabular}

be applied with good accuracy. Inspecting the results, it is apparent that the models agree quite well with each other during the summer months. They deviate from each other in the winter months, when the effect of the difference in the diffuse radiation parameterization is at its maximum. The RMSE results indicate that the anisotropic models (Hay and Klucher) show similar performance on an overall basis (within $5 \%)$ but the isotropic model exhibits much larger error. The MBE results show that the isotropic model substantially underpredicts the irradiance incident on an inclined surface, and the Klucher model considerably overpredicts irradiance incident on an inclined surface on an overall basis. Our findings confirm the observation that the Klucher model describes the irradiance on inclined planes more accurately than the isotropic model or Hay's model.
Due to lack of measured tilted surface solar radiation data, Klucher's model is employed to estimate the radiation incident on a collector surface from measured horizontal radiation. The results of these calculations are plotted against the angle of tilt for summer, winter and all year round intended use. A summary of the effects of tilt angle on solar irradiation for south facing surfaces is shown in Fig. 1 .

\subsection{Optical parameters of the encapsulation module}

Reflection losses produced in photovoltaic modules operating in real conditions in comparison with standard ones are commonly neglected or simplified to a value that does not take into account the module tilt angle. In some applications of interest such as building integration of photovoltaic modules, architectural criteria or other limitations frequently force modules to have tilt angles far from their optimal values. As a consequence, optical losses increase to non-negligible quantities [17].

The different encapsulation layers cause multiple reflections inside and among the slabs (see scheme in Fig. 2). The
Fig. 1: Annual variation in estimating average daily radiation for a flat plate collector facing south for a latitude of $50^{\circ}$, and ground reflectance of 0.2
Fig. 2: Ray tracing through the module layers 
abbreviation EVA stands for ethylene-vinyl-acetate, which serves as a mechanical, thermal and optical interface between the solar cells and the glass cover. ARC stands for the antireflective coating of the solar cell. It consists of $\mathrm{TiO}_{2}$ or another optical material to bridge over the refractive indices of silicon and the front cover. Refractive indices and transmissivity coefficients are given in Table 3 .

Table 3: Refractive indices and transmissivity coefficient for the wavelength of $\lambda=800 \mathrm{~nm} \mathrm{[5]}$

\begin{tabular}{|l|r|r|c|c|}
\hline Materials & $\begin{array}{c}\text { Refractive } \\
\text { index }\end{array}$ & Thickness & \multicolumn{2}{|c|}{$\begin{array}{c}\text { Transmissivity } \\
\text { coefficient }\end{array}$} \\
\hline & & & $\begin{array}{c}\text { Normal } \\
\text { incidence }\end{array}$ & $\begin{array}{c}\text { Incidence } \\
\text { at } 60^{\circ}\end{array}$ \\
\hline Glass & 1.526 & $3.2 \mathrm{~mm}$ & 0.951 & 0.941 \\
EVA & 1.450 & $0.6 \mathrm{~mm}$ & 0.992 & 0.990 \\
ARC & 1.700 & $50-60 \mathrm{~nm}$ & 1.000 & 1.000 \\
Silicon & 3.690 & $0.5 \mathrm{~mm}$ & - & - \\
\hline
\end{tabular}

Light rays are modeled as objects having the properties of electromagnetic waves $[1,15]$. Each ray can exist either as incident or as one of its reflected, refracted, absorbed and transmitted components, produced by the respective optical process (i.e., reflection, refraction, etc.). At the interfaces, a matrix method derived from electromagnetic theory is used to determine the probability of a photon being reflected, transmitted or absorbed in the interface layers. The calculation technique is based on the model of [19, 23], which calculates the radiation transfer properties of multi-layer structures. Their model was modified to generate an equivalent application in the domain of photovoltaic solar cell simulation.

\subsubsection{Radiation transmission through a photovoltaic module}

When light is incident upon the interface between two optically different materials, in general part of the light is reflected and part transmitted. If the medium on what is nominally chosen to be the outside (o) of the interface has a complex refractive index $n_{\mathrm{o}}$ and the medium on the inside (i) has a refractive index, $n_{\mathrm{i}}$ then, for a given wavelength, Snell's law states that:

$$
\theta_{\mathrm{o}}=\arcsin \left[\left(n_{\mathrm{i}} / n_{\mathrm{o}}\right) \sin \theta_{\mathrm{i}}\right] .
$$

For an incident wave of amplitude $E_{\mathrm{o}}^{+}$traveling across an interface from outside to inside (the superscript denotes the direction of propagation), the amplitude reflection coefficient $(r)$ and amplitude transmission coefficient $(t)$ are defined as:

$$
r_{\mathrm{oi}}=E_{\mathrm{o}}^{-} / E_{\mathrm{O}}^{+} ; \quad t_{\mathrm{oi}}=E_{\mathrm{i}}^{+} / E_{\mathrm{O}}^{+} .
$$

Similar expressions hold for waves traveling in the opposite direction, for which it can be shown that:

$$
r_{\mathrm{io}}=-r_{\mathrm{Oi}} ; \quad t_{\mathrm{oi}} t_{\mathrm{io}}-r_{\mathrm{oi}} r_{\mathrm{io}}=1 .
$$

An alternative approach is to calculate the transmission through the multi-layer using transfer matrices. Each matrix, in an ordered sequence of matrix multiplications, represents one of two possible transformations of the radiation fields. The transformations are due to:
- Transmission across an interface (interface matrix),

- Transmission through a layer (layer matrix).

The interface matrix: The definition of the interface $(M)$ follows from consideration of the boundary conditions at an interface. Let $E_{\mathrm{O}}^{+}$and $E_{\mathrm{i}}^{+}$represent the amplitudes of the net electric field propagation towards, and away from, an interface in the direction from the outside to the inside medium (Fig. 3). $E_{\mathrm{O}}^{-}$and $E_{\mathrm{i}}^{-}$are the corresponding net electric fields traveling in the reverse direction. $E_{i}^{+}$is then composed of the transmitted component of $E_{\mathrm{O}}^{+}$and the reflected component of $E_{\mathrm{i}}^{-}, E_{\mathrm{O}}^{-}$consists of the transmitted component of $E_{\mathrm{i}}^{-}$and the reflected component of $E_{\mathrm{O}}^{+}$. Equations (16) and (17) enable this to be expressed mathematically as:

$$
\begin{aligned}
& E_{\mathrm{i}}^{+}=t_{\mathrm{Oi}} E_{\mathrm{O}}^{+}+r_{\mathrm{iO}} E_{\mathrm{i}}^{-} \\
& E_{\mathrm{O}}^{-}=t_{\mathrm{iO}} E_{\mathrm{i}}^{-}+r_{\mathrm{Oi}} E_{\mathrm{O}}^{+}
\end{aligned}
$$

or

$$
\left[\begin{array}{c}
E_{\mathrm{O}}^{+} \\
E_{\mathrm{O}}^{-}
\end{array}\right]=\left[\begin{array}{cc}
\frac{1}{t_{\mathrm{Oi}}} & \frac{r_{\mathrm{oi}}}{t_{\mathrm{Oi}}} \\
\frac{r_{\mathrm{Oi}}}{t_{\mathrm{oi}}} & \frac{1}{t_{\mathrm{oi}}}
\end{array}\right] \cdot\left[\begin{array}{c}
E_{\mathrm{i}}^{+} \\
E_{\mathrm{i}}^{-}
\end{array}\right]=M_{\mathrm{oi}}^{(\mathrm{E})} \cdot\left[\begin{array}{c}
E_{\mathrm{i}}^{+} \\
E_{\mathrm{i}}^{-}
\end{array}\right]
$$

Fig. 3: The electric fields at an interface

When transmission across an interface is considered in terms of intensities, the following matrix equation is obtained:

$$
\left[\begin{array}{c}
I_{\mathrm{O}}^{+} \\
I_{\mathrm{O}}^{-}
\end{array}\right]=\left[\begin{array}{cc}
\frac{1}{\tau_{\mathrm{oi}}} & \frac{\Re_{\mathrm{oi}}}{\tau_{\mathrm{oi}}} \\
\frac{\Re_{\mathrm{oi}}}{\tau_{\mathrm{oi}}} & \frac{1}{\tau_{\mathrm{oi}}}
\end{array}\right] \cdot\left[\begin{array}{c}
I_{\mathrm{i}}^{+} \\
I_{\mathrm{i}}^{-}
\end{array}\right]=M_{\mathrm{oi}}^{(\mathrm{I})} \cdot\left[\begin{array}{c}
I_{\mathrm{i}}^{+} \\
I_{\mathrm{i}}^{-}
\end{array}\right] .
$$

The layer matrix: The layer matrix $(N)$ describes the attenuation of a wave as it traverses the medium within a layer. When a wave propagates in the positive direction between the interfaces of a thin layer, its initial amplitude $E_{\mathrm{f}}^{+}(1)$ within the layer is attenuated to, say, $E_{\mathrm{f}}^{+}(2)$ on reaching the second interface (Fig. 4). A wave of initial amplitude $E_{\mathrm{f}}^{-}$(2) traveling in the opposite direction through the layer is reduced to $E_{\mathrm{f}}^{-}(1)$. If the layer has thickness $d$ then from the definition of an attenuation factor,

$$
\begin{aligned}
& E_{\mathrm{f}}^{+}(2)=\Delta_{\mathrm{f}}(d) E_{\mathrm{f}}^{+}(1) \\
& E_{\mathrm{f}}^{-}(2)=\Delta_{\mathrm{f}}(-d) E_{\mathrm{f}}^{-}(1)
\end{aligned}
$$

or

$$
\left[\begin{array}{c}
E_{\mathrm{f}}^{+}(1) \\
E_{\mathrm{f}}^{-}(1)
\end{array}\right]=\left[\begin{array}{cc}
1 / \Delta_{\mathrm{f}}(d) & 0 \\
0 & \Delta_{\mathrm{f}}(d)
\end{array}\right] \cdot\left[\begin{array}{c}
E_{\mathrm{f}}^{+}(2) \\
E_{\mathrm{f}}^{-}(2)
\end{array}\right]=N_{\mathrm{f}}^{(\mathrm{E})} \cdot\left[\begin{array}{c}
E_{\mathrm{f}}^{+}(2) \\
E_{\mathrm{f}}^{-}(2)
\end{array}\right] \text {. }
$$

Matrix multiplication: The following equation represents the transformation of the electric fields by a thin layer 
Fig. 4: Transmission through a thin absorbing layer

(f) sandwiched between a pair of outer (o) and inner (i) media:

$$
\left[\begin{array}{c}
E_{\mathrm{O}}^{+} \\
E_{\mathrm{O}}^{-}
\end{array}\right]=M_{\mathrm{of}}^{(\mathrm{E})} \cdot N_{\mathrm{f}}^{(\mathrm{E})} \cdot M_{\mathrm{fi}}^{(\mathrm{E})} \cdot\left[\begin{array}{c}
E_{\mathrm{i}}^{+} \\
E_{\mathrm{i}}^{-}
\end{array}\right] .
$$

This involves the multiplication of three matrices, the first and third being interface materials, the second the layer matrix. A system is composed of a greater number of thin layers, say layers $1,2,3, \ldots, q$ sandwiched in the transformation.

$$
\begin{aligned}
{\left[\begin{array}{c}
E_{\mathrm{O}}^{+} \\
E_{\mathrm{O}}^{-}
\end{array}\right] } & =M_{\mathrm{o} 1}^{(\mathrm{E})} \cdot N_{1}^{(\mathrm{E})} \cdot M_{12}^{(\mathrm{E})} \cdot N_{2}^{(\mathrm{E})} \cdot \ldots \\
& \ldots \cdot N_{\mathrm{q}-1}^{(\mathrm{E})} \cdot M_{\mathrm{q}-1, \mathrm{q}}^{(\mathrm{E})} \cdot N_{\mathrm{q}}^{(\mathrm{E})} \cdot M_{\mathrm{qi}}^{(\mathrm{E})}\left[\begin{array}{c}
E_{\mathrm{i}}^{+} \\
E_{\mathrm{i}}^{-}
\end{array}\right] .
\end{aligned}
$$

Equation (24) can be use to obtain the total amplitude reflection and transmission coefficients, $\mathfrak{R}_{\mathrm{oi}}$ and $\tau_{\mathrm{oi}}$ of the multiple thin layer structure. This follows by considering the surrounding media to be of infinite thickness, so that there is no net reflected wave in medium (i) incident upon the inner interface (i.e., $E_{\mathrm{i}}^{-}=0$ ). By setting $E_{\mathrm{O}}^{+}=1$ Equation (24) becomes:

$$
\begin{aligned}
{\left[\begin{array}{c}
1 \\
\Re_{\mathrm{oi}}
\end{array}\right] } & =M_{\mathrm{o} 1}^{(\mathrm{E})} \cdot N_{1}^{(\mathrm{E})} \cdot M_{12}^{(\mathrm{E})} \cdot N_{2}^{(\mathrm{E})} \cdot \ldots \\
& \cdots \cdot N_{\mathrm{q}-1}^{(\mathrm{E})} \cdot M_{\mathrm{q}-1, \mathrm{q}}^{(\mathrm{E})} \cdot N_{\mathrm{q}}^{(\mathrm{E})} \cdot M_{\mathrm{qi}}^{(\mathrm{E})}\left[\begin{array}{c}
\tau_{\mathrm{oi}} \\
0
\end{array}\right]
\end{aligned}
$$

which can be solved for $\Re_{\mathrm{oi}}$ and $\tau_{\mathrm{oi}}$.

\subsubsection{Absorbed solar radiation}

The prediction of collector performance requires information on the solar energy absorbed by the collector absorber plate. The solar energy incident on a tilted collector can be found by the methods of Section 3.1. This incident radiation has three different spatial distributions: beam radiation, diffuse radiation, and ground reflected radiation, and each must be treated separately. Using Klucher's formulation, Equation (7) can be modified to give the absorbed radiation, $S$, by multiplying each term by the appropriate transmittance-absorptance product:

$$
\begin{aligned}
S & =(\tau \alpha)_{\mathrm{b}}\left(G_{\mathrm{H}}-I_{\mathrm{d}, \mathrm{H}}\right) R_{\mathrm{b}}+(\tau \alpha)_{\mathrm{d}} \cdot I_{\mathrm{d}, \mathrm{H}} \\
& \cdot\left[\frac{1+\cos \beta}{2}\left(1+F_{1} \sin ^{3} \frac{\beta}{2}\right)\left(1+F_{1} \cos ^{2} \theta \sin ^{3} \theta_{\mathrm{Z}}\right)\right]+ \\
+ & \frac{1}{2}(\tau \alpha)_{\mathrm{g}} G_{\mathrm{H}} \rho(1-\cos \beta) .
\end{aligned}
$$

The subscripts $b, d$, and $g$ represent beam, diffuse, and ground, respectively. Equation (27) can be used to find the proper absorptance:

$$
\begin{aligned}
\alpha & =1-\tau_{\mathrm{a}} \\
\tau_{\mathrm{a}} & =\frac{I_{\text {transmitted }}}{I_{\text {incident }}}=\exp \left(-\frac{K \cdot L}{\cos \theta_{\mathrm{O}}}\right)
\end{aligned}
$$

where $K$ is the proportionality constant, the extinction coefficient, which is assumed to be a constant in the solar spectrum. For glass, the value of $K$ varies from approximately $4 \mathrm{~m}^{-1}$ for "water white" glass (which appears white when viewed on the edge) to approximately $32 \mathrm{~m}^{-1}$ for poor (greenish cast of edge) glass. $L$ is the path length in the medium.

\subsubsection{Efficiency limits of photovoltaic modules}

The analytical results above were applied to analyze the optical behavior of an encapsulated photovoltaic solar cell. Photovoltaic modules have an angle of incidence AOI dependent optical behavior that can be measured and used to improve the analysis of array performance. Like absolute air mass, solar angle of incidence is time of day dependent. Its effect on the short circuit current of a photovoltaic module results from two causes.

The first is familiar to solar enthusiasts as the "cosine effect". The "cosine effect" is independent of the module design, and is only geometry related. Due to the cosine effect, the short circuit current from a module varies directly with the cosine of the AOI. For example, at AOI $=60^{\circ}$ the cosine effect reduces the short circuit current by one half compared to the normal incidence condition.

The second way in which the short circuit current is affected by the AOI is dependent on the module design. The optical characteristics of the module materials located between the sun and the solar cells cause the effect. Above $45^{\circ}$ incidence angle the reflectivity starts to increase, and reaches $20 \%$ losses at $75^{\circ}$ (see Fig. 5). The influence of a module's optical characteristics on its performance is shown in Fig. 6.
Fig. 5: Calculated relative transmission, reflection, and absorption of radiation for glass cover 


\subsection{Problem formulation}

The solution starts by assuming that the photovoltaic solar module is composed of three layers. For the purposes of the 3D-thermal model the solar module is divided into a number of meshes, each mesh having six faces, $(\mathrm{XY}=$ Top, $\mathrm{YX}=$ Bottom, $\mathrm{YZ}=$ Front, $\mathrm{ZY}=$ Back, $\mathrm{XZ}=$ Right and $\mathrm{ZX}=$ Left), $\mathrm{i}, \mathrm{j}, \mathrm{k}$ are the coordinates of the meshes. The computer code was written in Fortran. Simulation outputs allow a complete evaluation of the temperature distribution inside the photovoltaic module and can be of great interest both for system designers and for researchers. The technique used in this code involves calculating the temperature by an explicit method using iteration processes. The study is confined to a single silicon photovoltaic module of the following dimensions: thickness of silicon $0.5 \mathrm{~mm}$, sandwiched between a glass layer which is taken as $3 \mathrm{~mm}$ and Mylar $0.5 \mathrm{~mm}$, giving a total photovoltaic module thickness of $4 \mathrm{~mm}$. Both length and width are taken as $40 \mathrm{~mm}$. Also, it is assumed that all the heat is absorbed inside the silicon wafer homogeneously. The results are shown in Figs. 7 and 8, which show the temperature distribution along the XY-Plane at the mid Z-axis of the silicon wafer, and the temperature distribution along the

Fig. 6: Influence of angle of incidence on cell parameters XZ-Plane at mid Y-axis.

\section{Thermal performance of a photovoltaic module}

Solar radiation irradiating at the surface of the solar module is absorbed partially by various materials of the module. Almost all radiation is absorbed in the silicon. Only a very small part is absorbed in other materials of the solar module. This will produce heat inside the solar cell. There are heat loss mechanisms from the front and rear surface of the module: free convection, wind convection and radiative heat loss. Inside cell heat is transmitted by conduction. The equations describing the heat flows within the cell are given below:

Radiation heat transfer equation: The total radiative heat flow from the module, $Q_{\text {rad }}[\mathrm{W}]$ to the environment comprises the components of the radiation exchange of the front and rear sides of the module with the sky and the ground. These components are also functions of the module surface temperature, $T_{(\mathrm{i}, \mathrm{j}, \mathrm{k})}$, ambient air temperature, $T_{\infty}$ and the relevant emissivity $\varepsilon$ also; these components are determined by the so-called radiation shape factor, $F_{\mathrm{s}}$ the radiative surface area of the module, $A_{\mathrm{m}}$ and the Stefan-Boltzmann constant $\delta$. In general this can be expressed as [11]:

$$
Q_{\mathrm{rad}}=F_{\mathrm{s}} \varepsilon \delta \mathrm{A}_{\mathrm{m}}\left(T_{(\mathrm{i}, \mathrm{j}, \mathrm{k})}^{4}-T_{\infty}^{4}\right)
$$

Convection heat transfer equation: Convective heat flows, $Q_{\text {conv. }}$ cannot be treated in a closed mathematical model and, therefore have to be computed by iteration or approximation. The convective heat transfer coefficient $h_{\infty}\left[\mathrm{W} / \mathrm{m}^{2} \mathrm{~K}\right]$ is a bulky function of the actual air temperature and module surface temperature, air viscosity, thermal conductivity and heat capacity, and finally wind speed. The models used are based on the following equation:

$$
Q_{\text {conv. }}=h_{\infty} A_{\mathrm{c}}\left(T_{(\mathrm{i}, \mathrm{j}, \mathrm{k})}-T_{\infty}\right) .
$$

Conduction heat transfer equation: can be given as:

$$
Q_{\text {cond. }}=\frac{K_{\mathrm{th}} A_{\mathrm{m}}}{h}\left(T_{(\mathrm{i}, \mathrm{j}, \mathrm{k})}-T_{(\mathrm{i}+1, \mathrm{j}, \mathrm{k})}\right) \text {. }
$$




\section{For the meshes at the corners of the cell}

For $\mathrm{i}=1, \mathrm{j}=1, \mathrm{k}=1$

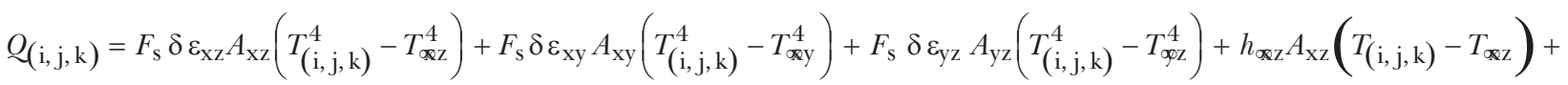

$$
\begin{aligned}
& +h_{\text {xy }} A_{\mathrm{xy}}\left(T_{(\mathrm{i}, \mathrm{j}, \mathrm{k})}-T_{\mathrm{xy}}\right)+h_{90 \mathrm{z}} A_{\mathrm{yz}}\left(T_{(\mathrm{i}, \mathrm{j}, \mathrm{k})}-T_{\mathrm{goz}}\right)+K_{\mathrm{th}} \frac{A_{\mathrm{zx}}}{h_{\mathrm{y}}}\left(T_{(\mathrm{i}, \mathrm{j}, \mathrm{k})}-T_{(\mathrm{i}, \mathrm{j}+1, \mathrm{k})}\right)+ \\
& +K_{\mathrm{th}_{\mathrm{m}}} \frac{A_{\mathrm{yx}}}{h_{\mathrm{z}}}\left(T_{(\mathrm{i}, \mathrm{j}, \mathrm{k})}-T_{(\mathrm{i}, \mathrm{j}, \mathrm{k}+\mathrm{l})}\right)+K_{\mathrm{th}} \frac{A_{\mathrm{zy}}}{h_{\mathrm{x}}}\left(T_{(\mathrm{i}, \mathrm{j}, \mathrm{k})}-T_{(\mathrm{i}+1, \mathrm{j}, \mathrm{k})}\right)
\end{aligned}
$$

For the meshes at the edges of the cell

For $\mathrm{i}=1, \mathrm{k}=1, \mathrm{j}=2, \ldots,\left(n_{\mathrm{y}}-1\right)$

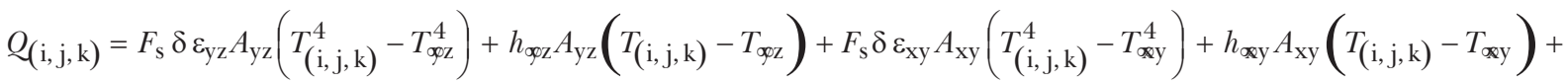

$$
\begin{aligned}
& +K_{\mathrm{th}_{\mathrm{m}}} \frac{A_{\mathrm{yx}}}{h_{\mathrm{z}}}\left(T_{(\mathrm{i}, \mathrm{j}, \mathrm{k})}-T_{(\mathrm{i}, \mathrm{j}, \mathrm{k}+\mathrm{l})}\right)+K_{\mathrm{th}_{\mathrm{m}}} \frac{A_{\mathrm{zy}}}{h_{\mathrm{x}}}\left(T_{(\mathrm{i}, \mathrm{j}, \mathrm{k})}-T_{(\mathrm{i}+1, \mathrm{j}, \mathrm{k})}\right)+K_{\mathrm{th}_{\mathrm{m}}} \frac{A_{\mathrm{xz}}}{h_{\mathrm{y}}}\left(T_{(\mathrm{i}, \mathrm{j}, \mathrm{k})}-T_{(\mathrm{i}, \mathrm{j}+1, \mathrm{k})}\right)+ \\
& +K_{\mathrm{th}} \frac{A_{\mathrm{zx}}}{h_{\mathrm{y}}}\left(T_{(\mathrm{i}, \mathrm{j}, \mathrm{k})}-T_{(\mathrm{i}, \mathrm{j}-1, \mathrm{k})}\right)
\end{aligned}
$$

\section{For the meshes inside the cell body}

For $\mathrm{i}=2, \ldots,\left(n_{\mathrm{x}}-1\right), \mathrm{j}=2, \ldots,\left(n_{\mathrm{y}}-1\right), \mathrm{k}=2, \ldots,\left(n_{\mathrm{z}}-1\right)$

$$
\begin{aligned}
Q_{(\mathrm{i}, \mathrm{j}, \mathrm{k})}= & K_{\mathrm{th}} \frac{A_{\mathrm{xy}}}{h_{\mathrm{z}}}\left(T_{(\mathrm{i}, \mathrm{j}, \mathrm{k})}-T_{(\mathrm{i}, \mathrm{j}, \mathrm{k}-\mathrm{l})}\right)+K_{\mathrm{th}} \frac{A_{\mathrm{yx}}}{h_{\mathrm{z}}}\left(T_{(\mathrm{i}, \mathrm{j}, \mathrm{k})}-T_{(\mathrm{i}, \mathrm{j}, \mathrm{k}+\mathrm{l})}\right)+K_{\mathrm{th}} \frac{A_{\mathrm{zy}}}{h_{\mathrm{x}}}\left(T_{(\mathrm{i}, \mathrm{j}, \mathrm{k})}-T_{(\mathrm{i}-1, \mathrm{j}, \mathrm{k})}\right)+ \\
& +K_{\mathrm{th}_{\mathrm{m}}} \frac{A_{\mathrm{yz}}}{h_{\mathrm{x}}}\left(T_{(\mathrm{i}, \mathrm{j}, \mathrm{k})}-T_{(\mathrm{i}+\mathrm{l}, \mathrm{j}, \mathrm{k})}\right)+K_{\mathrm{th}_{\mathrm{m}}} \frac{A_{\mathrm{xz}}}{h_{\mathrm{y}}}\left(T_{(\mathrm{i}, \mathrm{j}, \mathrm{k})}-T_{(\mathrm{i}, \mathrm{j}-1, \mathrm{k})}\right)+K_{\mathrm{th}_{\mathrm{m}}} \frac{A_{\mathrm{zx}}}{h_{\mathrm{y}}}\left(T_{(\mathrm{i}, \mathrm{j}, \mathrm{k})}-T_{(\mathrm{i}, \mathrm{j}+1, \mathrm{k})}\right)
\end{aligned}
$$

\section{For the meshes at the surface}

$$
\begin{aligned}
\text { For } \mathrm{i} & =2, \ldots,\left(n_{\mathrm{x}}-1\right), \mathrm{j}=2, \ldots,\left(n_{\mathrm{y}}-1\right), \mathrm{k}=1 \\
Q_{(\mathrm{i}, \mathrm{j}, \mathrm{k})} & =F_{\mathrm{s}} \delta \varepsilon_{\mathrm{xy}} A_{\mathrm{xy}}\left(T_{(\mathrm{i}, \mathrm{j}, \mathrm{k})}^{4}-T_{\mathrm{xxy}}^{4}\right)+h_{\mathrm{xxy}} A_{\mathrm{xy}}\left(T_{(\mathrm{i}, \mathrm{j}, \mathrm{k})}-T_{\mathrm{x} \mathrm{y}}\right)+K_{\mathrm{th}} \frac{A_{\mathrm{yx}}}{h_{\mathrm{z}}}\left(T_{(\mathrm{i}, \mathrm{j}, \mathrm{k})}-T_{(\mathrm{i}, \mathrm{j}, \mathrm{k}+\mathrm{l})}\right)+ \\
& +K_{\mathrm{th}_{\mathrm{m}}} \frac{A_{\mathrm{yz}}}{h_{\mathrm{x}}}\left(T_{(\mathrm{i}, \mathrm{j}, \mathrm{k})}-T_{(\mathrm{i}-1, \mathrm{j}, \mathrm{k})}\right)+K_{\mathrm{th}} \frac{A_{\mathrm{zy}}}{h_{\mathrm{x}}}\left(T_{(\mathrm{i}, \mathrm{j}, \mathrm{k})}-T_{(\mathrm{i}+1, \mathrm{j}, \mathrm{k})}\right)+K_{\mathrm{th}_{\mathrm{m}}} \frac{A_{\mathrm{zx}}}{h_{\mathrm{y}}}\left(T_{(\mathrm{i}, \mathrm{j}, \mathrm{k})}-T_{(\mathrm{i}, \mathrm{j}-1, \mathrm{k})}\right)+ \\
& +K_{\mathrm{th}} \frac{A_{\mathrm{xz}}}{h_{\mathrm{y}}}\left(T_{(\mathrm{i}, \mathrm{j}, \mathrm{k})}-T_{(\mathrm{i}, \mathrm{j}+1, \mathrm{k})}\right)
\end{aligned}
$$

For the meshes at the gap

$$
\begin{aligned}
Q_{(\mathrm{i}, \mathrm{j}, \mathrm{k})} & =K_{\mathrm{th}} \frac{A_{\mathrm{yz}}}{h_{\mathrm{x}}}\left(T_{(\mathrm{i}, \mathrm{j}, \mathrm{k})}-T_{(\mathrm{i}-\mathrm{l}, \mathrm{j}, \mathrm{k})}\right)+K_{\mathrm{th}} \frac{A_{\mathrm{zy}}}{h_{\mathrm{x}}}\left(T_{(\mathrm{i}, \mathrm{j}, \mathrm{k})}-T_{(\mathrm{i}+\mathrm{l}, \mathrm{j}, \mathrm{k})}\right)+ \\
& +K_{\mathrm{th}_{\mathrm{m}}} \frac{A_{\mathrm{xz}}}{h_{\mathrm{y}}}\left(T_{(\mathrm{i}, \mathrm{j}, \mathrm{k})}-T_{(\mathrm{i}, \mathrm{j}+1, \mathrm{k})}\right)+K_{\mathrm{th}} \frac{A_{\mathrm{zx}}}{h_{\mathrm{y}}}\left(T_{(\mathrm{i}, \mathrm{j}, \mathrm{k})}-T_{(\mathrm{i}, \mathrm{j}-1, \mathrm{k})}\right)+ \\
& +K_{\mathrm{th}_{\mathrm{m}}} \frac{A_{\mathrm{xy}}}{h_{\mathrm{z}}}\left(T_{(\mathrm{i}, \mathrm{j}, \mathrm{k})}-T_{(\mathrm{i}, \mathrm{j}, \mathrm{k}-\mathrm{l})}\right)+h_{\mathrm{gap}} A_{\mathrm{yx}}\left(T_{(\mathrm{i}, \mathrm{j}, \mathrm{k})}-T_{(\mathrm{i}, \mathrm{j}, \mathrm{k}+1)}\right)
\end{aligned}
$$

Where:

$h_{\mathrm{x}}=$ width of mesh in $\mathrm{x}, h_{\mathrm{y}}=$ width of mesh in $\mathrm{y}, h_{\mathrm{z}}=$ width of mesh in $\mathrm{z}, A_{\mathrm{xz}}=h_{\mathrm{x}} h_{\mathrm{z}}, A_{\mathrm{xy}}=h_{\mathrm{x}} h_{\mathrm{y}}, A_{\mathrm{yz}}=h_{\mathrm{y}} h_{\mathrm{z}}, m=$ material number, $n_{\mathrm{x}}=$ total No. of meshes in x-direction, $n_{\mathrm{y}}=$ total No. of meshes in y-direction and $n_{\mathrm{z}}=$ total No. of meshes in z-direction.

\subsection{Temperature dependence of solar module parameters}

Fig. 9 shows the dependence of $I_{\mathrm{sc}}, V_{\mathrm{oc}}, F F$, and $P_{\mathrm{m}}$ on temperature. As the cell temperature increases, the short circuit current, $I_{\mathrm{sc}}$ somewhat increases, and the maximum power, $P_{\mathrm{m}}$ the open circuit voltage, $V_{\mathrm{oc}}$ and the fill factor, $F F$ decrease. The short circuit current increase with temperature is attributed to light absorption variation. Under the conditions considered, the solar absorption edges shift due to a decrease in the crystal forbidden gap width, and the number of pairs generated in the bulk increases. Alternatively, as the temperature increases, the intrinsic light absorption factor changes and the short circuit current increases. To explain the variation of $V_{\mathrm{oc}}$ with temperature, the effects of all voltage controlling parameters (mobility, lifetime, energy band gap and absorption coefficient) have to be included in the analysis. In the general case, open circuit voltage is determined by the relation $V_{\mathrm{oc}} \approx \ln \left(I_{\mathrm{sc}} / I_{\mathrm{d}}\right)$, by which $V_{\mathrm{oc}}$ must grow as $I_{\mathrm{sc}}$ increases. Depending on the current flowing mechanism, the 
Fig. 9: Variation of key parameters of a silicon solar cell at high temperatures

value of $I_{\mathrm{d}}$ is proportional to $n_{\mathrm{i}}^{2}$. As the temperature increases $n_{\mathrm{i}}$ grows exponentially and causes exponential growth of $I_{\mathrm{d}}$. Moreover, the crystal forbidden gap narrowing with temperature increase leads to an increase in the dark current, $I_{\mathrm{d}}$ and minimizes the positive effect of the absorption factor. As a result, $\mathrm{V}_{\mathrm{oc}}$ decreases. The maximum power across the optimal load and the efficiency depend strongly on temperature; the shape of this dependence is close to linear.

\section{Conclusions}

A simple and accurate close-form solution for the optical and thermal behaviors of a solar cell array were investigated by simulation and by some verifying experiments, in order to obtain more accurate results in predicting the actual performance of photovoltaic modules, without resorting to a lengthy, time-consuming iterative solution which has to be repeated for any change in the parameters of the array.

\section{Nomenclature}

$G_{\mathrm{H}} \quad$ Hourly global radiation incident on a horizontal surface, $\mathrm{kWh} / \mathrm{m}^{2} /$ day

$G_{\mathrm{T}} \quad$ Hourly global radiation incident on an inclined surface, $\mathrm{kWh} / \mathrm{m}^{2} /$ day

$I_{\mathrm{b}, \mathrm{H}} \quad$ Hourly beam radiation incident on an inclined surface, $\mathrm{kWh} / \mathrm{m}^{2} /$ day

$I_{\mathrm{b}, \mathrm{T}} \quad$ Hourly beam radiation incident on a horizontal surface, $\mathrm{kWh} / \mathrm{m}^{2} /$ day

$I_{\mathrm{d}, \mathrm{H}} \quad$ Hourly sky diffuse radiation incident on a horizontal surface, $\mathrm{kWh} / \mathrm{m}^{2} /$ day

$I_{\mathrm{d}, \mathrm{T}} \quad$ Hourly sky diffuse radiation incident on an inclined surface, $\mathrm{kWh} / \mathrm{m}^{2} /$ day

$I_{\mathrm{o}} \quad$ Extraterrestrial hourly radiation incident on a horizontal surface, $\mathrm{kWh} / \mathrm{m}^{2} /$ day

$I_{\mathrm{g}, \mathrm{H}} \quad$ Hourly ground reflected radiation incident on a horizontal surface

$I_{\mathrm{g}, \mathrm{T}} \quad$ Hourly ground radiation incident on an inclined surface, $\mathrm{kWh} / \mathrm{m}^{2} /$ day

$I_{\mathrm{T}, \text { cir }} \quad$ Circumsolar radiation component

$I_{\mathrm{T}, \text { iso }}$ Isotropic radiation component
Refractive index

$A_{\mathrm{I}} \quad$ Anisotropic index

$R_{\mathrm{b}} \quad$ The ratio of beam radiation on the tilted surface to that on a horizontal surface

$r \quad$ Reflection of incident

$\beta \quad$ Surface slope, degrees

$\delta \quad$ Declination, degrees

$\phi \quad$ Latitude, degrees

$\rho \quad$ Ground albedo

$\theta_{1} \quad$ Incidence angle, the angle between beam radiation and the vertical, degrees

$\theta_{\mathrm{o}} \quad$ Angles of refraction

$\omega \quad$ Sunrise hour angle, degrees

$\gamma \quad$ Surface azimuth angle, degrees

$E_{\mathrm{O}}^{-}, E_{\mathrm{i}}^{+}$Amplitudes of the reflected and transmitted waves

$\Re \quad$ Reflectance

$Q_{\text {rad }}$ Total radiative heat flow from the module, W

$Q_{\text {conv }}$ Convective heat flow, W

$Q_{\text {cond }}$ Quantity of heat transfer by conduction from one mesh to another, $\mathrm{W}$

$K_{\text {th }} \quad$ Thermal conductivity of different materials of solar cell, $\mathrm{W} / \mathrm{mK}$

$T_{(\mathrm{i}, \mathrm{j}, \mathrm{k})}$ Module surface temperature, ${ }^{\circ} \mathrm{C}$

$T_{\infty} \quad$ Ambient temperature, ${ }^{\circ} \mathrm{C}$

$\varepsilon \quad$ Emissivity

$F_{\text {s }} \quad$ Radiation shape factor

$A_{\mathrm{m}} \quad$ Radiative surface area of the module

$A_{\mathrm{c}} \quad$ The surface area of the cell, $\mathrm{m}^{2}$

\section{References}

[1] Born, M., Wolf, E.: Principles of optics. $5^{\text {th }}$ Edition, Pergamon, New York, 1975

[2] Bugler, J.: The determination of hourly insolation on a tilted plane using a diffuse irradiance model based on hourly measured global horizontal insolation. Solar Energy, 1977, Vol. 19, No. 5, pp. 477

[3] Dave, J.: Validity of the isotropic distribution approximation in solar energy estimations. Solar Energy, 1977, Vol. 19, pp. 331

[4] Duffie, J., Beckman, W.: Solar engineering of thermal processes. Wiley, New York, 1980

[5] Fraidenraich, N., Vilela, O.: Exact solutions for multiplayer optical structures: Application to PV modules. Solar Energy, 2000, Vol. 69, No. 5, pp. 357

[6] Gardner, C., Nadeau, C.: Estimating south slope irradiance in the Arctic - A comparison of experimental and modeled values. Solar Energy, 1988, Vol. 41, pp. 227

[7] Green, M.: High efficiency silicon solar cells. Trans Tech Publications, Switzerland, 1987

[8] Gueymard, C.: An anisotropic solar irradiance model for tilted surfaces and its comparison with selected engineering algorithms. Solar energy, 1987, Vol. 38, p. 367

[9] Hay, J.: Calculation of monthly mean solar radiation for horizontal and tilted surfaces. Solar Energy, 1979, Vol. 23, p. 301 
[10] Hay, J., Davies, J.: Calculation of the solar radiation incident on an inclined surface. Proceedings First Canadian Solar Radiation Data Workshop, 1980, p. 59

[11] Holman, J.: Heat Transfer. Mc Graw Hill, 1982

[12] Hottel, H. C., Woertz, W.: Performance of flat plate solar heat collectors. Transactions of American Society of Mechanical Engineers, 1942, Vol. 64, pp. 91

[13] Ineichen, P., Perez, R., Seals, R.: The importance of correct albedo determination for adequately modeling energy received by tilted surfaces. Solar Energy, 1987, Vol. 39, pp. 221

[14] Iqbal, M.: An Introduction to solar radiation. Academic Press, Toronto, 1983

[15] Klein, M., Furtak, T.: Optics. Wiley, New York, 1993

[16] Klucher, T.: Evaluation of models to predict insolation on tilted surfaces. Solar Energy, 1979, Vol. 23, p. 114

[17] Martin, N.: Comparative study of the angular influence on different PV module technologies. Proceeding $14^{\text {th }}$ E.C. PVSEC, 1997

[18] Nahumura, S.: Applied Numerical Methods with Software. Prentice Hall, New York, 1991

[19] Perommer, P., Lomas, K., Seale, C., Kupke, C.: The radiation transfer through coated and tinted glazing. Solar Energy, 1995, Vol. 54, No. 5, p. 287
[20] Smith, G.: Numerical solution of partial differential equations: Finite Difference Method. Oxford, 1979

[21] Temps, R. C., Coulson, K. L.: Solar radiation incident upon slopes of different orientations, Solar Energy, 1977, Vol. 19, p. 179

[22] Wenham, S., Green, M., Watt, M.: Applied photovoltaics. University of New South Wales, Center of Photovoltaic Systems and Devices, Australia, 1994

[23] Harbeke, B.: Coherent and Incoherent Reflection and Transmission of Multi-layer Structures. Applied Physics, 1989, Vol. 39, pp. 165-170

Eng. Hamdy Kamal Elminir e-mail: ehamdy@hotmail.com phone: +42022435 2212 fax: +420224353949

Prof. Ing. Vítězslav Benda, CSc. Ing. Ivan Kudláček, CSc.

Czech Technical University in Prague Faculty of Electrical Engineering

Technická 2, 16627 Praha 6, Czech Republic 